\title{
(4) Development in Diagnostic Logic
}

\section{Contribution of the Medical Databank}

\author{
Yasushi TAtsuzawa, M. D. \\ Department of Internal Medicine, School of Medicine, Keio University
}

Doctors, originally simple faith curers, are now on their way to becoming scientist-physicians, and will proceed to develop social-scientist-physicians. One problem that confronting them is the increasing amount of medical information, the resulting specialization of medical fields and increasing number of specialists as well as paramedicals. No adequate information processing system has been developed to meet the informational age except for information processing system such as Medical Literature Analysis and Retrieval System (MEDLARS). Under such conditions, the Medical Databank is necessary to organize and efficiently process. the large amount of original data prior to publication.

One of the functions of the Medical Databank is to store recorded abstracts from academy meetings. The information retrieval system, Japanese Medical Abstracts Scanning System (JAMASS), which has been developed by the International Medical Information Center (IMIC) in cooperation with the Japanese Society of Neurology since 1970, is one system enabling information retrieval instead of reference retrieval in case of MEDLARS. The program also enables one to obtain a concrete grasp of comprehensive structure of medical information. Furthermore, the system can provide secondary feedback function of clarified structure of medical information to medical education.

The recorded abstracts of academy meetings can be retraced to the original medical data. The IMIC found difficulties in the attempt to process the data of discharge abstracts of the Hamamatsu Red Cross Hospital, namely, difficulties due to medical care system, habits of filling out a Karte and information storage system of hospitals in Japan. At this point, it has been shown that the criteria of diagnostic logic of medicine have not been based on optimality under omniscient rationality of natural science, but rather on satisfying under adaptive rationality of behavioural science, that is, gradual resolution of problems leads to a high level of satisfaction. On the other hand, with the development of natural science doctors gradually became scientist-physicians requiring diagnostic logic based on omniscient rationality. However, in order for the scientist-physician to gain this diagnostic logic they need preconditions such as high evaluating, predictive and penetrating abilities. These preconditions are satisfied by the Medical Databank which store vast amount of information required to base diagnostic logic on omniscient rationality. Until now, 
diagnostic logic has been forced to depend on adaptive rationality because of the scant amount of information even though some problems with consistency of standardization and objectiveness remained, because medicine has an obligation to needs of society.

At present, medicine has reached the second stage of development to organized medicine, with the transfer of its logical base from adaptive rationality to omniscient rationality. Therefore, the Medical Databank is expected to act as intermediary in this process. Diagnostic logic based on omniscient rationality orients to the establishment of mechanical diagnosis, however, at the present medical conditions with the limited evaluating, predictive and penetrating abilities, there is a large gap between information storage and actual decision making by computers. Herein, the responsibility for diagnosing arises, demonstrating one of reasons why a doctor is indispensable in diagnosis. It also shows that despite recent development in information science, abilities of computers are still limited.

For the Medical Databank to contribute to the improvement of medical treatment, not only medical information in a narrow sense but the entire spectrum of information related to medical treatment, such as hospital administration and furthermore, regional medical care programs should be collected. In addition, an information databank network dealing with other social problems should be established. This would provide doctors with broad consciousness and steady in-

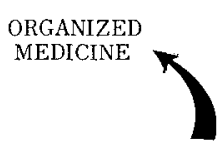

DIAGNOSTIC LOGIC BASED ON OMNISCIENT RATIONALITY formation base, allowing them to

DIAGNOSTIC LOGIC BASED ON ADAPTIVE RATIONALITY

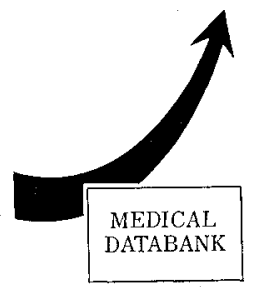

Fig. 1 consider the happiness of mankind, giving rise to social-scientist-physicians.

In brief, the Medical Databank may be defined in the following manner.

The Medical Databank which collects, stores and processes information concerning medicine or health in a broad sense, may be used by all qualified people. It is of help in judging, evaluating and planning in a medical field. At the same time, the Medical Databank is oriented towards clarifying the relationships between the various characteristics of the information or data of medicine, and towards grasping comprehensively and standardizing medicine. The Medical Databank aims at a combined type, comprehensive information system, independently administered, but with the support of society. Finally the Medical Databank aims at promoting the transfer of the logical base of medical diagnosis from adaptive rationality to omniscient rationality, and at developing medicine from its present state to organized medicine. 